\title{
Research on Wood Cell Shape Analysis Methods based on Fourier Descriptors
}

\author{
Lei Zhao 1 ,a and Jian Hua Wang ${ }^{2, b}$ \\ ${ }^{1}$ Heilongjiang International University, Harbin 150025, China \\ ${ }^{2}$ Harbin normal University, Harbin 150025, China

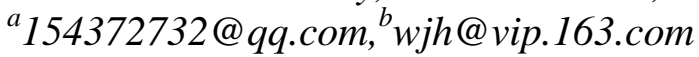

\begin{abstract}
The shape parameter of wood microscopic cell is one of the important parameters in the identification of wood species. Fourier Descriptors is often used to describe the boundaries of closed areas. The paper analyzes the slice images of five kinds of common wood cells, extracting the mathematical parameters of the cells, obtaining the average value and simulating the benchmark cell shape of the five wood species to provide data support for subsequent recognition experiments.
\end{abstract}

Keywords: Fourier Descriptors, slice images, cell shape, wood species

\section{Introduction}

The recognition of object shape is a very important research topic in the field of pattern recognition and image processing. There are mountains of application cases in the fields such as image processing, machine vision and target-analysis recognition etc., [1-5]. There are a variety of forms for describing the shape of the object on the computer, for example, coordinates of the boundary curves, the distance between boundary points and the center of the object and the boundary points' tangential direction and curvature. There are different algorithms for different expression of shapes, such as methods based boundary features, invariant moment features, regression models, Fourier descriptors, etc., [6-12]. The study has found that it is quite easy for human beings to identify the outline of target objects while it is rather difficult for the computer. The exact and rapid object recognition is still a rather hard job for machines when the target object are translated, zoomed, rotated arbitrarily, twisted or sheltered. Here are a few common methods for the description of shapes.

\subsection{Boundary Feature based Method}

This method utilizes boundary features description to obtain relevant shape parameters of images. Boundary length; the boundary global features have been taken into consideration with this method. It is the length of the contour of the target object. Curvature, a pixel's slope difference on both sides of the curve is the so-called curvature. The curvature reflects the curving degree of the boundary. Hough transformation; the basic idea is the duality of points and lines. As the target object contour pixels are low, it can be connected together to form a closed contour by way of the overall features of the image. Boundary histogram method; First find image contour edges by differential image, and then draw a histogram of edge magnitude and direction, generally constructing gray matrix. 


\subsection{Geometric Parameter Method}

The method is used for simply expressing and matching shapes by regional characteristics description. Geometric parameter method generally includes the relevant feature parameters such as eccentricity of the target object, the object solid degree, the long axis of the target object, the object contour perimeter, the target area and target tightness and etc. Generally, for content and image based query system, the mathematical parameters such as target object quasi-circle degree, eccentricity of objects, the algebraic invariant moment and principal axis direction are often used to identify and classify images based on shape feature. In particular, image segmentation and processing are the prerequisites for the parameter extraction and are closely related to the accuracy of the parameters. Therefore, shape parameter can not be acquired from images of poor segmentation [13-20].

\subsection{Shape Invariant Moment Method}

The shape invariant moment method is commonly used in the process of $2 \mathrm{D}$ graphics recognition and classification. Torque is a linear feature unchanging with the scaling, rotation and translational motion of objects. Therefore, regional characteristics of images can be expressed with this method.

\subsection{Topology Description Method}

Topology is usually used to study the nature of graphics. These properties are generally not affected by changes of the images, unless the images are torn up or blocked. It is generally used to describe the general characteristics of objects [21-24].

\subsection{The Method of Fourier Descriptors}

Fourier descriptors are often used to describe the boundary of enclosed areas. Algorithm is to describe object boundary contour with Fourier descriptors, transforming two-dimensional problems into a one-dimensional periodic problem by using the properties of closure and periodicity. However, this method is not applicable for composite enclosed contours. The three shape expressions derived by the boundary points are respectively curvature function, centroid distance and complex coordinate function [25-27].

\section{Fourier Descriptors}

\subsection{The basic Principles of Fourier Descriptors}

Fourier descriptors are the results of frequency domain analysis for the target object boundary contour as well as Fourier transform coefficients of the object boundary contour curve. The research shows that Fourier descriptors have different conversion results for the target object's shape, scale, orientation, etc. Therefore, normalization processing for Fourier descriptors shall be carried out before the use of them to describe the shape of the target object. Through experimental comparison, it can be easily found that Fourier descriptors have better recognition accuracy for object description in a variety of typical analytic methods. Moreover, the discrete Fourier transform is a reversible operation, so there is no loss of information in the process of transformation.

Fourier descriptors algorithm can be described as follows: Assuming that the contour of the target cell is a closed curve, and there is a point named $P(l)$ while the coordinates of this 
point meet $x(l)+j y(l)$, then this can be understood as periodic function with the period of cell girth. It can be described by Fourier series expansion.

In the Fourier series, there is a close relationship between the shape of the object boundary contour curve and $z(k)$. Here it is called Fourier descriptors. When the coefficients are enough, the shape of the object can be fully expressed by Fourier descriptors, and can be restored.

Assuming the coordinates of the target object contour coordinates can be described as follows: $\{x(n), y(n) \mid n=0,1 \ldots \ldots, N-1\}$. Then the plural expression is:

$$
z(n)=x(n)+j y(n), n=0,1, \ldots \ldots, N-1\}
$$

The boundary curve of object contour can be expressed in the form of a one-dimensional space after conversion. For closed contour curve, this sequence has cyclic characteristic, and the cycle is $\mathrm{N}$.

A one-dimensional Discrete Fourier Transformation (DFT) is defined as follows:

$$
Z(k)=\sum_{n=0}^{N-1} z(n) \exp \left(-\frac{j 2 \pi k n}{N}\right),(0 \leq n \leq N-1)
$$

The Inverse transformation formula is:

$$
z(n)=\frac{1}{N} \sum_{n=0}^{N-1} Z(k) \exp \left(\frac{j 2 \pi k n}{N}\right),(0 \leq n \leq N-1)
$$

The experiment shows that Fourier coefficients are so sensitive to contour's low frequency that few coefficients can distinct various shapes. However, Fourier descriptors directly obtained are particularly sensitive to the information like displacement, so that normalized operation is quite necessary here.

\subsection{The Invariance of Fourier Descriptors}

Only by understanding the influence of the translation, selection, scale and other conversions to the Invariance of Fourier descriptors can we make Fourier descriptors invariant with the conversions and use certain operation methods to avoid the influence.

(1)The influence of translation

If the target contour curve shift $z_{0}$

$$
z_{t}(n)=z(n)+z_{0}
$$

Then $Z(0)$ changing

$$
\begin{gathered}
Z(k)=\sum_{n=0}^{N-1} z_{t}(n) \exp \left(-\frac{j 2 \pi k n}{N}\right) \\
=\sum_{n=0}^{N-1} z(n) \exp \left(-\frac{j 2 \pi k n}{N}\right)+\sum_{n=0}^{N-1} z_{0}(n) \exp \left(-\frac{j 2 \pi k n}{N}\right)
\end{gathered}
$$

The latter is the sum of a geometric series, with only the Fourier coefficient

$$
Z_{t}(0)=Z(0)+z_{0}
$$


(2)The influence of rotation

If the curve rotates $\theta$,

$$
z_{r}(n)=z(n) \exp (j \theta)
$$

The Fourier coefficients produce the equivalent shift.

$$
Z_{r}(k)=Z(k) \exp (j \theta)
$$

(3)The influence of scale

If a target object woks a times telescopic fold on the origin of the curve,

$$
z_{s}(n)=a z(n)
$$

The Fourier coefficients' amplitude produces equivalent shift.

$$
Z_{s}(k)=a Z(k)
$$

(4) The influence of starting point on Fourier coefficients

If the position of the starting point of the curve is changed,

$$
z_{s p}(n)=z\left(n-n_{0}\right)
$$

Then the Fourier coefficients' modulation will be generated.

$$
Z_{s p}(k)=Z(k) \exp \left(-\frac{j 2 \pi k n_{0}}{N}\right)
$$

That is, the K Fourier coefficient $Z(k)$ is multiplied by a coefficient $\exp \left(-\frac{j 2 \pi k n_{0}}{N}\right)$.

The above research proves that the shape information expressed by Fourier descriptors is invariance in certain aspects. Coefficient amplitude $\mathrm{P} Z(k) \mathrm{P}, k=0,1, \ldots \ldots, N-1$ has nothing to do with curve starting position, so it has the property of translational and selective invariance, but $Z(0)$ is special which does not have the property of translation invariance. Phase information $\arg (Z(k)), k=0,1, \ldots \ldots, N-1$ has the property of scale invariance. According to these properties, one can only use amplitude value of the Fourier coefficients, which is to carry out normalization of Fourier coefficients. The approach is: Let $Z(0)=0$, and then $\mathrm{P} Z(k) \mathrm{P}$ has the property of selected translation invariance; If each efficient $\mathrm{P} Z(k) \mathrm{P}$ is divided by $\mathrm{P} Z(1) \mathrm{P}, \frac{\mathrm{P} Z(k) \mathrm{P}}{\mathrm{P} Z(1) \mathrm{P}}$ do not change with scale, and $\frac{\mathrm{P} Z(k) \mathrm{P}}{\mathrm{P} Z(1) \mathrm{P}}$ will have the properties of selection, translation and scale invariance at the same time. In this paper, this is expressed as Fourier descriptors to express shape information. 


\subsection{Fourier Descriptor with Polygon Approximation}

The shape information of the object is described by pixel coordinates $p_{0}, p_{1}, \ldots, p_{N}$ in order to track the contour of the target object. For the purpose to reduce redundancy characteristic points and to further reduce the computational workload and noise, the boundary points extracted by polygonal approximation algorithms are the approximate representations of the original contour curves under a given approximate accuracy of D (typically 1-2 pixels). Because of the existence of approximate accuracy, the number of polygonal feature points after approximation is much less than that of the original image, so that it greatly reduces the amount of storage and computation.

Chen and $\mathrm{Su}$ polygonal approximation algorithm is to extract the maximum curvature point on the curve as an approximation point of the polygon. The realization process of the algorithm is as follows:

(1) Initialization. Set $p_{0}$ as the starting point and extract characteristic values of all the subsequent points

set $i=0$; set $j=i+2$; set $p_{0}$ is the very first point to observe.

(2) Distance Value. Calculate the distance value between the point in any interval $\left(p_{i}, p_{j}\right)$ in any point of the profile curve and linear segment $p_{i} p_{j}$ and calculate the maximum $p_{m}$.

if $j>N$ then stop

Point $p_{m}$, then $d(i, j, m)=\max _{i<k<j} d(i, j, k)$

(3) Determine whether $p_{m}$ is the featured point needed

if $\left(d\left(i, j, k^{\prime}\right)>D \quad\right.$ then set $p_{m}$ as the next feature point;

set $\mathrm{i}=\mathrm{m} ;$ set $\mathrm{j}=\mathrm{i}+2$;

$\operatorname{goto}(2)$;

else $\mathrm{j}=\mathrm{j}+1$; goto $(2)$

Here,

$$
d(i, j, m)=\left|\frac{\left(y_{j}-y_{i}\right) x_{m}-\left(x_{j}-x_{i}\right) y_{m}+\left(y_{i} x_{j}-x_{i} y_{j}\right)}{\left(\left(x_{j}-x_{i}\right)^{2}+\left(y_{j}-y_{i}\right)^{2}\right)^{1 / 2}}\right|
$$

it means the distance between $p_{m}$ and $p_{i} p_{j}$.

\subsection{The Rapid Fast Fourier Transform (FFT) Method}

Normally, it is necessary to make discretion for target contour curves to get the Fourier descriptors. Numerous points can be obtained through discretion and these points have the feature of discrete equal interval. Equation (14) shows FFT coefficient of discrete points. The coefficients can be used as the description of the shape.

$$
\left\{\begin{array}{c}
z(k)=\sum_{n=0}^{N-1}(x(n)+i y(n)) e^{-j \frac{2 \pi}{N} k n} \\
p(n)=\frac{1}{N} \sum_{k=0}^{N-1} z(k) e^{j \frac{2 \pi}{N} k n}
\end{array}\right.
$$


The discretization of the original boundary curve is a priority to FFT, and the discretization is of equal chord. Here in order to ensure accuracy of the expression of object shape, it is recommended to increase the number of sampling points, which results in the large amount of calculation of Fourier change. The discretization of the target contours lowers the accuracy, so it is necessary to reduce the sampling point so as to improve the speed. FFT can be replaced by continuous Fourier transform in each line segment through polygonal approximation method of the target object boundary curve. The experiment shows that the method not only retains the original shape information, but also reduces the amount of computation, and effectively eliminates calculation errors caused by discrete sampling. Calculation method is as follows:

$$
\begin{aligned}
& p(l)=x(l)+j(l)=\sum_{k=-\infty}^{+\infty} z(k) e^{j \frac{2 \pi k l}{N}} \\
& z(k)=\frac{1}{L} \int_{0}^{L} p(l) e^{j \frac{2 \pi k l}{N}} d l=\frac{1}{L} \sum_{n=0}^{N-1} \int_{l_{n}}^{l_{n+1}} p(l) e^{j \frac{2 \pi k l}{N}} d l= \\
& \frac{1}{L} \sum_{n=0}^{N-1} \int_{l_{n}}^{l_{n+1}}\left\lfloor x_{n}+\left(l-l_{n}\right) \frac{x_{n+1}-x_{n}}{l_{n+1-l_{n}}}+j\left(y_{n}+\left(l-l_{n}\right) \frac{y_{n+1}-y_{n}}{l_{n+1}-l_{n}}\right) \mid e^{j \frac{2 \pi k l}{N}} d l=\right. \\
& \left\{\quad \frac{1}{L} \sum_{n=0}^{N-1} \frac{l_{n+1}-l_{n}}{2}\left[\left(x_{n+1}-x_{n}\right)+j\left(y_{n+1}-y_{n}\right)\right], k=0\right. \\
& \left\{\frac { 1 } { j 2 \pi k } \sum _ { n = 0 } ^ { N - 1 } \left\{\left[x_{n}+j y_{n}+\left(\frac{x_{n+1}-x_{n}}{l_{n+1}-l_{n}}+j \frac{y_{n+1}-y_{n}}{l_{n+1}-l_{n}}\right)\left(\frac{L}{j 2 \pi k}-l_{n}\right)\right]\left(e^{-j \frac{2 \pi k}{L} l_{n}}-e^{-j \frac{2 \pi k}{L} l_{n+1}}\right)+\right.\right. \\
& \left.\left(\frac{x_{n+1}-x_{n}}{l_{n+1}-l_{n}}+j \frac{y_{n+1}-y_{n}}{l_{n+1}-l_{n}}\right)\left(l_{n} e^{-j \frac{2 \pi k}{L} l_{n}}-l_{n+1} e^{-j \frac{2 \pi k}{L} l_{n+1}}\right)\right\}, k \neq 0
\end{aligned}
$$

The coordinates of the vertex $p_{n}$ is $\left(x_{n}, y_{n}\right) . l_{n}$ means the sum of length of sides from the polygon vertex $p_{n}$ to $p_{0}$. L is the perimeter of a polygon.

\subsection{The Normalization of Fourier Descriptors}

Fourier descriptor is related to many parameters, such as scales of target shape etc, so the normalization of Fourier descriptors is to recognize the shape information of translation, rotation and invariant scale. In order to retain the phase position and mode information of Fourier descriptors, and to recognize the translation and invariant scale of any shape, all the observed target objects can be rotated to the same direction and position, then the new initial point position order can be formed from the point of insertion between object boundary and the positive $\mathrm{x}$-axis, and Fourier descriptor $\frac{\mathrm{P} Z(k) \mathrm{P}}{\mathrm{P} Z(1) \mathrm{P}}$ is used for shape recognition. The disadvantage of this method is the calculation of the position of the starting point and the rotation of the shape to the same direction, which needs a large amount of calculation. This paper presents a relatively simple Fourier descriptors normalization method.

$$
d^{\prime}(k) \equiv \frac{z(k)}{e^{j \frac{2 \pi}{L}(k-1) a} z(1)}
$$




$$
e^{j \frac{2 \pi}{L} a}=\frac{z(1) e^{-j \varphi}}{\|z(1)\|}, k=1,2, \ldots, N-1
$$

Here principal orientation of a shape is set as a variable $\varphi$.

After the above process, the normalized Fourier descriptors keep modulus and phase characteristics of Fourier variation factor and the feature of the size and direction of the shape curve of the target object unrelated to the initial point. It effectively avoids losing phase information due to normalization and improves the accuracy of object shape recognition.

\subsection{The Determination of the Principal Orientation of a Shape}

The main direction of the general contour of an object is usually located on the straight line through the center of gravity and with an inclination of $\beta$. After KL transformation of the shape, $\beta$ meets the direction of characteristic vector with the largest characteristic value.

$$
\beta=\tan ^{-1} \frac{u_{02}-u_{20}+\sqrt{\left(u_{02}-u_{20}\right)^{2}+4 u_{11}^{2}}}{2 u_{11}}
$$

Here, $u_{20}, u_{02}, u_{11}$ are the $(p+q)$ order center distance of the enclosed area. The $(p+q)$ order center distance of the area surrounded by contour curve of the object can be calculated by the vertex of object contour approximated polygon.

There are two options for the straight line of the direction angle of an object contour curve, i.e., forward $\beta$ and reverse $\beta+\pi$. One way is determined by the third moment of a shape, to rotate the shape a degree of $-\beta$, so the main direction of the shape is the direction of the part with relatively less energy.

$$
\varphi=\left\{\begin{array}{c}
\beta, u_{30} \leq 0 \\
\beta+\pi, u_{30}>0
\end{array}\right.
$$

\subsection{The Calculation of Euclidean Distance}

Here we can use the normalized Fourier descriptor $d^{\prime}(k)$ to compare the resemblance degree between any two shapes, I and J, and recognize the shape of the contour curve of the target object with the selection, translation and scale invariance. Euclidean distance can be used to indicate the difference between shapes as the components of the Fourier descriptors are all orthogonal. The experiment shows that the high-frequency component of the Fourier transformation is particularly sensitive to noise, so Fourier descriptors are generally only used to describe the difference of shapes in the low-frequency parts. When Distance $=0$, it means that two objects have the same shape information. The greater distance represents the greater difference between shapes.

$$
D \text { is } \tan c e=\sqrt{\sum_{k=2}^{M}\left\|d_{I}^{\prime}(k)-d_{J}^{\prime}(k)\right\|^{2}}
$$

The paper uses the digital curve discrete polygon approximation method, replacing discrete Fourier transformation with continuous Fourier transformation to calculate the Fourier descriptors of shapes. The method can not only reduce the error caused by equal interval 
sampling discretization of the object boundary contour curve etc, but also reduces the amount of computation. In this paper, the principal direction of the shape is utilized to eliminate the effect of the initial point position, which reduces the loss of phase information. The paper also provides normalized Fourier descriptors which can keep modulus and phase information simultaneously.

\section{The Shape Matching Experiment of Wood Benchmark Cells}

The so-called shape matching is to measure the similarity degree among different shapes [28-31] in accordance with certain criteria. It analyses the shape characteristics of the cells through the shape matching of the wood cell and mathematic shape. In order to make mathematical analysis for particular wood cell, the concept of benchmark cell has been put forward. The benchmark cell is to analyze lots of wood cell microscopic images, extract various mathematical parameters of cells and obtain the average value, which can reflect the features of most cells of each wood cell image.

The shape of a benchmark cell should be the average of most cell shapes, so its shape should represents the characteristics of the shape of this wood species. Therefore, in the simulation of a benchmark cell, the very first thing is to determine the basic contour of cells, and then analyze the number of triangles, quadrangles, pentagons and hexagons, to use the shape found most frequently as a benchmark cell.

The following experimental analysis is carried out with real wood cells as target samples. The results are shown in Table 1 and Figure 1.

In the experiment, the shapes of the five cells of five wood species in Table 6-1 are analyzed respectively. The cell image is substantially the six prismatic shapes through mountains of microscopic observation of cell slices as is introduced in previous sections. Therefore, in the experiment, the triangles, quadrangles, pentagons and hexagons in Figure 61 are selected as testing samples to analyze the number of the cells approximate to hexagons among the wood species. The results are shown in Table 2.

From the experiment data in Table 2, the approximate shape analysis results of single cell of the five wood species can be obtained, as shown in Table 3.

From the above experimental results, it can be seen that the benchmark cell can be simulated by using $d^{\prime}(k)$ to make approximate shape analysis of the cells in the experimental samples, and thus to simulate the benchmark cell shape. The project selects 10 microscopic wood cell images of each of the five wood species, the resolution of which is $1392 * 1040$, and the analysis results are shown in Table 4.

The experimental data in Table 4 shows that the cell shapes of five wood species are mostly pentagons and hexagons, respectively accounting for $94.3 \%, 90.4 \%, 87.9 \%, 90.2 \%$ and $89.5 \%$ of total cells. Compared with artificial methods, the error rates of triangles, squares, pentagons and hexagons are shown in Table 5. From Table 5, it can be found that when using the method presented in this paper to calculate the number of triangular cedar cells, the maximum statistical error is $2.54 \%$ and an average error of shape analysis of each wood species is $0.69 \%$, smaller than the threshold value of $1 \%$. Therefore, the method is reliable for wood shape analysis.

\section{Conclusion}

The paper analyzes the main shape of the microscopic images of five coniferous wood species by using the Fourier descriptors method to implement cell shape approximation. The 
shape found most frequently is used as the benchmark cell. The experiment result shows that the method is reliable for wood shape analysis, with lower error value and better robustness.

Table 1. The Single Cell Image of Five Kinds of Wood Species

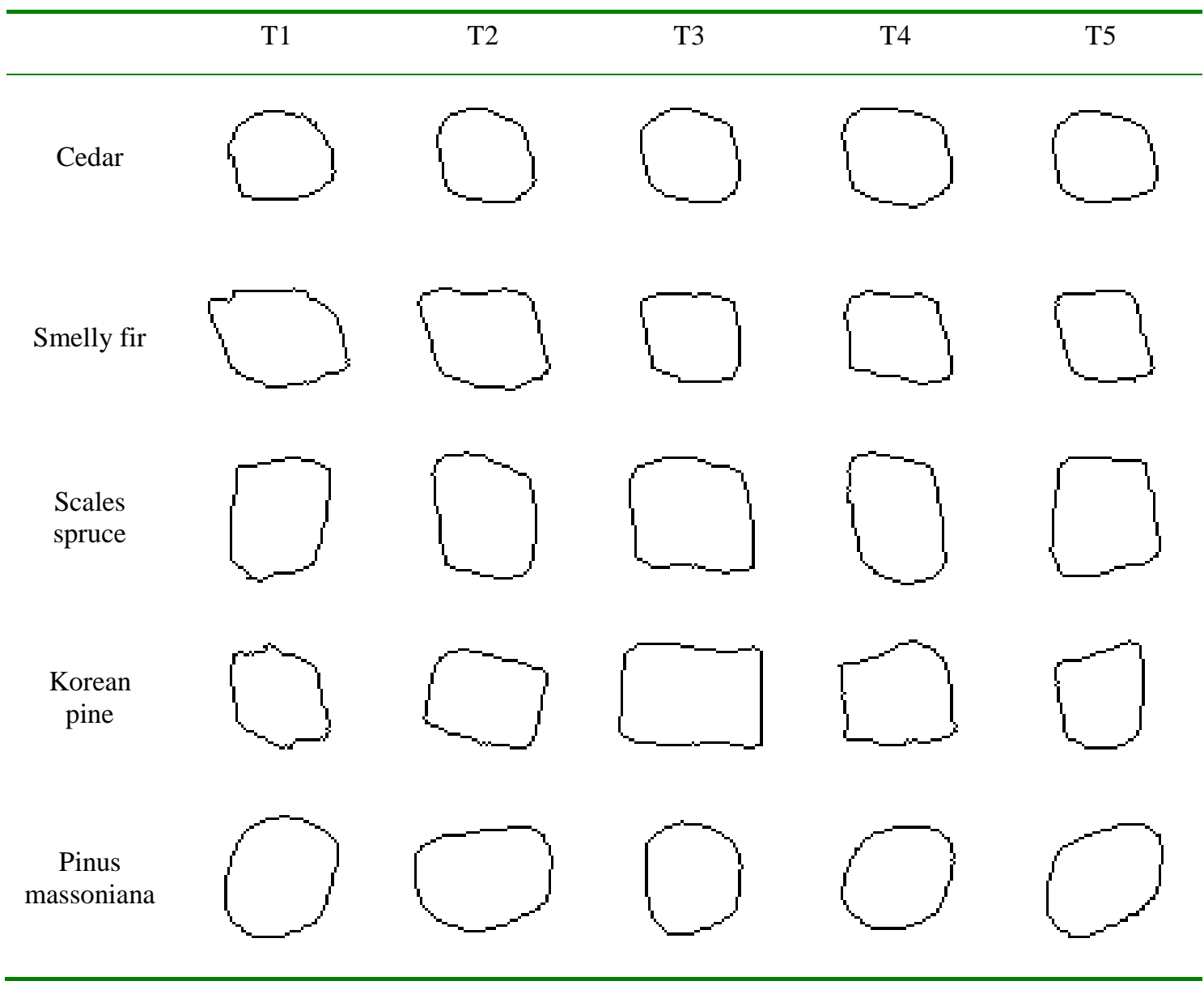

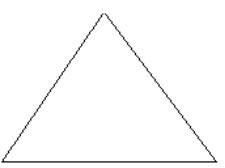

(a) triangle

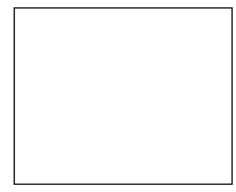

(b) quadrilatera

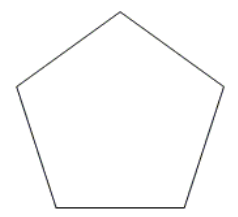

(c) pentagon

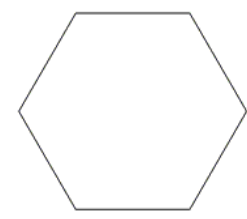

(d) hexagon

Figure 1. Testing Sample Images 
Table 2. Five Kinds of Wood Species Cell Images Adopting $d^{\prime}(k)$ to make Approximate Analysis with Testing Image

\begin{tabular}{|c|c|c|c|c|c|c|}
\hline $\begin{array}{l}\text { Wood } \\
\text { names }\end{array}$ & Test shape & $\mathrm{T} 1$ & $\mathrm{~T} 2$ & $\mathrm{~T} 3$ & $\mathrm{~T} 4$ & $\mathrm{~T} 5$ \\
\hline \multirow{4}{*}{ Cedar } & triangle & 1.002102933 & 0.937886556 & 0.976238298 & 0.994097062 & 0.931791547 \\
\hline & quadrilateral & 0.416995227 & 0.462308869 & 0.521599769 & 0.573717918 & 0.669541244 \\
\hline & pentagon & 0.324811690 & 0.416172852 & 0.476601484 & 0.570542103 & 0.666934552 \\
\hline & hexagon & 0.377897085 & 0.397128652 & 0.454308003 & 0.540976045 & 0.679923589 \\
\hline \multirow{4}{*}{ Smelly fir } & triangle & 0.991092775 & 0.861813205 & 0.879287982 & 0.862964269 & 0.933546239 \\
\hline & quadrilateral & 0.461732294 & 0.712642929 & 0.547377567 & 0.503707334 & 0.876294276 \\
\hline & pentagon & 0.453851356 & 0.776254258 & 0.489638708 & 0.522242458 & 0.889149966 \\
\hline & hexagon & 0.469567902 & 0.768508454 & 0.482768463 & 0.512735461 & 0.898092151 \\
\hline \multirow{4}{*}{$\begin{array}{l}\text { Scales } \\
\text { spruce }\end{array}$} & triangle & 0.734377106 & 0.692050364 & 0.606668397 & 0.709399646 & 0.696078863 \\
\hline & quadrilateral & 0.373588526 & 0.375078673 & 0.364002535 & 0.507706166 & 0.360227743 \\
\hline & pentagon & 0.351968913 & 0.356451948 & 0.444189305 & 0.494276823 & 0.390131763 \\
\hline & hexagon & 0.364983083 & 0.313070875 & 0.414351849 & 0.495386451 & 0.310783051 \\
\hline \multirow{4}{*}{$\begin{array}{c}\text { Korean } \\
\text { pine }\end{array}$} & triangle & 0.818991939 & 0.818991939 & 0.750222113 & 0.734521909 & 0.761486166 \\
\hline & quadrilateral & 0.775344015 & 0.775344015 & 0.436744049 & 0.470463648 & 0.530319316 \\
\hline & pentagon & 0.820803556 & 0.820803556 & 0.401898809 & 0.538983126 & 0.520004948 \\
\hline & hexagon & 0.815625574 & 0.815625577 & 0.370992993 & 0.466577282 & 0.542512191 \\
\hline \multirow{4}{*}{$\begin{array}{c}\text { Pinus } \\
\text { massoniana }\end{array}$} & triangle & 0.722811861 & 0.692050364 & 0.769570887 & 0.768819343 & 0.693621453 \\
\hline & quadrilateral & 0.345438644 & 0.375078673 & 0.425559895 & 0.644546619 & 0.391204786 \\
\hline & pentagon & 0.294075055 & 0.356451948 & 0.336473155 & 0.626706439 & 0.443705329 \\
\hline & hexagon & 0.264309851 & 0.313070876 & 0.274142429 & 0.657911634 & 0.454666032 \\
\hline
\end{tabular}

Table 3. Five Kinds of Wood Species Cell Approximate Shape Analysis Results

\begin{tabular}{cccccc}
\hline & T1 & T2 & T3 & T4 & T5 \\
\hline Cedar & pentagon & hexagon & hexagon & hexagon & pentagon \\
Smelly fir & pentagon & quadrilateral & hexagon & hexagon & quadrilateral \\
Scales spruce & pentagon & hexagon & quadrilateral & pentagon & hexagon \\
Korean pine & quadrilateral & quadrilateral & hexagon & hexagon & pentagon \\
$\begin{array}{c}\text { Pinus } \\
\text { massoniana }\end{array}$ & hexagon & hexagon & hexagon & pentagon & quadrilateral \\
\hline
\end{tabular}


Table 4. The Experiment Results of Five Wood Species

\begin{tabular}{cccccccccc}
\hline & \multicolumn{1}{c}{ This paper method } & \multicolumn{5}{c}{ Artificial recognition } \\
\hline & $\begin{array}{c}\text { The } \\
\text { number } \\
\text { of cells }\end{array}$ & $\begin{array}{c}\text { The } \\
\text { number } \\
\text { of } \\
\text { triangle }\end{array}$ & $\begin{array}{c}\text { The } \\
\text { number of } \\
\text { quadrangle }\end{array}$ & $\begin{array}{c}\text { The } \\
\text { number } \\
\text { of } \\
\text { pentagon }\end{array}$ & $\begin{array}{c}\text { The } \\
\text { number } \\
\text { of } \\
\text { hexagon }\end{array}$ & $\begin{array}{c}\text { The } \\
\text { number } \\
\text { of } \\
\text { triangle }\end{array}$ & $\begin{array}{c}\text { The } \\
\text { number of } \\
\text { quadrangle }\end{array}$ & $\begin{array}{c}\text { The } \\
\text { number } \\
\text { of } \\
\text { pentagon }\end{array}$ & $\begin{array}{c}\text { The } \\
\text { number } \\
\text { of } \\
\text { hexagon }\end{array}$ \\
\hline $\begin{array}{c}\text { Cedar } \\
\text { Smelly fir }\end{array}$ & 14762 & 384 & 457 & 3882 & 10039 & 394 & 465 & 3882 & 10021 \\
$\begin{array}{c}\text { Scales } \\
\text { spruce }\end{array}$ & 8057 & 209 & 595 & 2935 & 4647 & 208 & 585 & 2935 & 4658 \\
$\begin{array}{c}\text { Korean } \\
\text { pine } \\
\begin{array}{c}\text { Pinus } \\
\text { massoniana }\end{array}\end{array}$ & 6064 & 244 & 643 & 2447 & 5730 & 247 & 646 & 2450 & 5721 \\
\hline
\end{tabular}

Table 5. Error Rate

\begin{tabular}{crrrr}
\hline & $\begin{array}{c}\text { The deviation } \\
\text { of triangle }\end{array}$ & $\begin{array}{c}\text { The deviation of } \\
\text { quadrangle }\end{array}$ & $\begin{array}{c}\text { The deviation } \\
\text { of pentagon }\end{array}$ & $\begin{array}{c}\text { The deviation } \\
\text { of } \\
\text { hexagon }\end{array}$ \\
\hline Cedar & $2.54 \%$ & $1.72 \%$ & $0.00 \%$ & $0.18 \%$ \\
$\begin{array}{c}\text { Smelly fir } \\
\text { Scales }\end{array}$ & $0.48 \%$ & $1.71 \%$ & $0.00 \%$ & $0.24 \%$ \\
spruce & $0.00 \%$ & $1.53 \%$ & $0.37 \%$ & $0.45 \%$ \\
$\begin{array}{c}\text { Korean } \\
\text { pine }\end{array}$ & $1.21 \%$ & $0.46 \%$ & $0.12 \%$ & $0.16 \%$ \\
$\begin{array}{c}\text { Pinus } \\
\text { massoniana }\end{array}$ & $2.16 \%$ & $0.00 \%$ & $0.26 \%$ & $0.28 \%$ \\
\hline
\end{tabular}

\section{Acknowledgements}

This work is supported by the key laboratory for intelligence education and information engineering at Hei Longjiang province of China (No. 1155xnc107)

\section{References}

[1] L. Yong, "The research of essence recognition method based on gray level co-occurrence matrix of wood digital image", Nan Jing forestry university, (2010).

[2] H.D. Cheng and X. H. Jiang, "Color image segmentation based on homogram threshoding and region merging,PattemRecognition", vol. 35, no. 2, (2002), pp. 373-393.

[3] H. K. Hybrid, "Image Seglnentation Using Watersheds and Fast Region Merging", IEEE Transaetions on Image Proeessing, vol. 7, no. 12, (1998), pp. 16-24.

[4] B. Rockel and S. Lautner, "Lignin distribution in wood cell walls determined by TEM andbackscattered SEM techniques", Journal of Structural Biology, vol.143, (2003), pp. 77-84.

[5] J. Eitelberger and K. Hofstetter, "Prediction of transport properties of wood below the fiber saturation point a multiscale homogenization approach and its experi- mental validation", Part II Steady state moisture diffusion coefficient, Comp -os Sci Technol, vol. 71, no. 1, (2011), pp. 4551. 
[6] A. Rosenkilde and J. Arfvidsson, "Measurements and evaluation of moisture trans- port coefficients during drying of wood", Holzforschung, vol. 51, no. 4, (2007), pp. 372 -380.

[7] M. Peura , M. Mullera and R. Serimaa, " Structural studies of single wood cell walls by synchrotron X-ray microdiffraction and polarised light microscopy”, Nuclear Instruments and Methods in Physics Research, vol. 238, (2005), pp. 16-20.

[8] H. Qing and L. Mishnaevsky, "Moisture-related mechanical properties of softwood, 3D micromechanical modeling Hai Qing, Leon Mishnaevsky", Computational Materials Science, vol. 46, (2009), pp. 310-320.

[9] D.W. Stahle and P.T. Mushove, "Management implications of annual growth rings in Pterocarpus angolensis from Zimbabwe”, Forest Ecology and Management, vol. 124, (1999), pp. 217-229.

[10] J. Eitelberger, K. Hofstetter and S.V. Dvinskikh, "A multi-scale approach for simulation of transient moisture transport processes in wood below the fiber saturation point", Composites Science and Technology, vol. 71, (2011), pp.1727-1738.

[11] Z. Z. Jie and W. R. Sheng, " The Reaserch which Based on the shape of the contour feature extraction and recognition method", Computer engineering and application, vol.14, no. 3, (2006), pp. 92-94.

[12] X. Xue-qiang, W. A. Bo and H. Peng, "United Hu Moment and Their Application to TV Image Recogniton. Computer engineering and application”, vol. 29, (2006), pp. 213-215.

[13] C. X. Chun, "A Method for Shape Recognition", Pattern recognition and artificial intelligence, vol. 19, no. 6, (2006), pp. 758-763.

[14] Q. Hengnian and S. Tao, J. Shui, "Leaf characteristics based computer-aided plant identification model", Journal of zhejiang forestry college, vol. 20, no. 3, (2006), pp. 281-284.

[15] H. L. Frandsen, "Modeling of moisture transport in wood, state of the art andanalytic discussion, Tech. rep., Dept. of Building Technology and StructuralEngineering", Aalborg University, (2005).

[16] H. Ling, K. Okada, Smith, E. Landis and M. Gong, "Fracture and Fatigue in Wood, Wiley", (2003).

[17] S. C. Huang and Y. N. Sun, "PolygonalApproximationusingGenetieAlgorithmS", opattern Recognit- ion, vol. 32, (1999), pp. 409-1420.

[18] E. MIlios and E. G. M. Petrakis, "Shape retrieval based on dynamic programing", IEEE Trans image process, vol. 9, no. 1, (2000), pp. 141-146.

[19] B. I. Hassel, P. Berard, C. S. Moden and L. A. Berglund, "The single cube apparatus for shear testing fullfield strain data and finite element analysis of wood in transverse shear", Compos Sci Technol, vol. 69, (2009), pp. 77-82.

[20] A. Manceau, M.L. Schlegel, M. Musso, V.A. Sole, C. Gauthier, P.E.Petit, F. Trolard and Geochim, "Spectrochim", Acta 64 (2000), pp. 36-43.

[21] T. Ohtani, K. Kamasaki and C. Tanaka, "On abrasive wear property during three-body abrasion of wood, Wear", vol. 255, (2003), pp. 60-66.

[22] Q. H and E. R. Hancock, "Graph matching and clustering using spectral partitions", Pattern Recognition, vol. 39, (2006), pp. 22-34.

[23] Y. Chunping, Q. Bin, and I. Fei, "Priority Characteristics Definition of Graphic Elements for Graph Outline Extraction and its Applications", Journal of computer aided design and graphics, vol. 22, no. 1, (2010), pp. 44-49.

[24] F. Demirei, A. Shokoufandeh and Diekinson, "Skeletal shape abstraction from examples", PatternAna, Maeh, Intell, vol. 31, no. 5, (2009), pp. 944-952.

[25] A. M. Bronstein, M. M. Bronstein and A.M. Bruekstein, "Partial similarity of objects, or how to compare a centaur to a horse", Int.Joumal of ComPuter Vision, vol. 84, (2009), pp. 163-183.

[26] "Seasonal growth zones in the wood of Acacia karroo Hayne, their definition and implications", Commonwealth For. Rev. vol. 73, pp. 121-127.

[27] M. Yang , K. Kidiyo and R. Joseph , “A survey of shape feature extraction techniques”, Pattern Recognition. INTECH, vol. 169, (2010), pp. 106-115.

[28] J. Krepkowski, "Growth dynamics and potential for cross-dating and multi-century climate reconstr uction of Podocarpus falcatus in Ethiopia", dendrochronologia, vol. 30, (2012), pp. 257-265.

[29] H. E. Shuang-xi, Z. Shao-jun, X. Jing-liang and Y. Chun-yan, "Extracting profile feature points by a method based on arc length", Photoelectric engineering, vol. 31, no. 11, (2004), pp. 59-62.

[30] J. ZHENG, L. Zhengwen, M. Zhaorui and P. Jiluan, "Extraction of outline feature points based on the $\mathrm{m}$ inim um approach error", Journal of tsinghua university, vol. 48, no. 2, (2008), pp. 166-168.

[31] X. Feng, "Comparative study of boundary feature description methods for similar figure", Shu Zou University, (2006). 


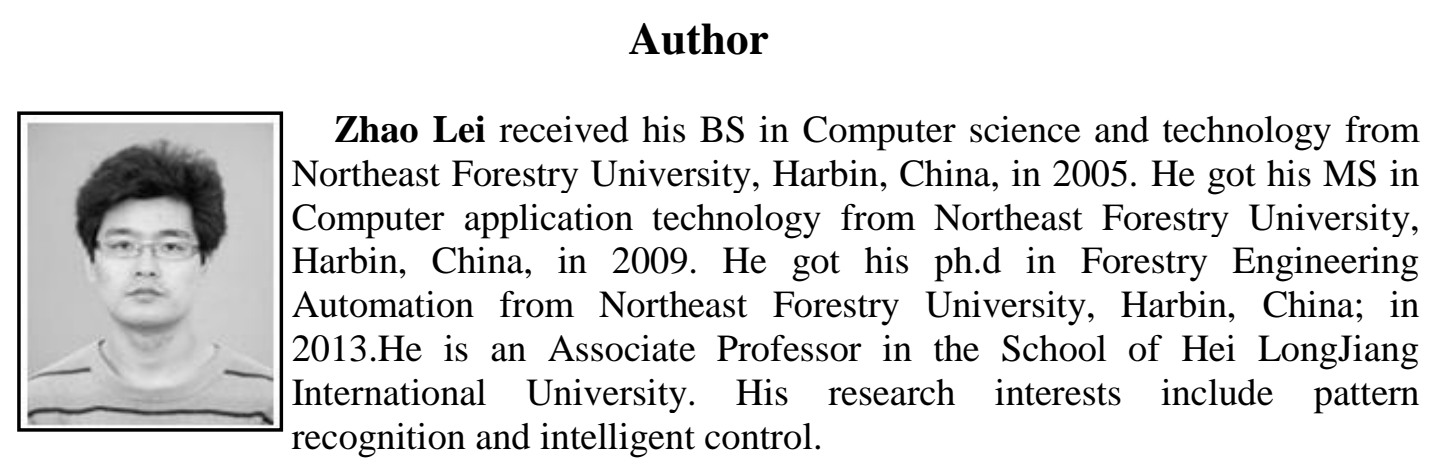


International Journal of Multimedia and Ubiquitous Engineering Vol. 10, No. 2 (2015) 